\title{
O uso da tecnologia assistiva por terapeutas ocupacionais no contexto educacional brasileiro: uma revisão da literatura ${ }^{1}$
}

\author{
Paloma Greicy Ferreira de Sousa ${ }^{a}$, Andrea Perosa Saigh Jurdi', \\ Carla Cilene Baptista da Silva ${ }^{b}$
}

aUniversidade Federal de São Paulo - UNIFESP, São Paulo, SP, Brasil.

${ }^{\text {bC }}$ urso de Terapia Ocupacional, Universidade Federal de São Paulo - UNIFESP, Santos, SP, Brasil.

\begin{abstract}
Resumo: Este artigo tem por objetivo apresentar, por meio de uma revisão bibliográfica, como os terapeutas ocupacionais têm se organizado para promover discussões sobre o uso dos recursos de tecnologia assistiva no ambiente escolar. Desse modo, foi realizada busca ativa acerca deste tema em diferentes bases de dados: Scientific Eletronic Library Online (Scielo); Biblioteca Virtual em Saúde (Bireme); Revista de Terapia Ocupacional da Universidade de São Paulo, e Cadernos de Terapia Ocupacional da Universidade Federal de São Carlos, no período de 2005 ao primeiro trimestre de 2013. Como resultado da busca, apresentou-se a sistematização de 11 estudos relacionados à tecnologia assistiva, à terapia ocupacional, ao contexto educacional e à inclusão escolar. A análise dos artigos mostrou que terapeutas ocupacionais se preocupam com a necessidade de formação de professores para o uso dos recursos de tecnologia assistiva no processo de escolarização de alunos com deficiência. O número de trabalhos encontrados ainda é incipiente frente à demanda que se apresenta na área de tecnologia assistiva e educação. Há um número maior de trabalhos referentes a relatos de pesquisa, poucos trabalhos sobre a fundamentação teórica da tecnologia assistiva e a sua relação com a terapia ocupacional no ambiente escolar.
\end{abstract}

Palavras-chave: Inclusão Educacional, Equipamentos de Autoajuda, Terapia Ocupacional.

\section{The use of assistive technology by occupational therapists in the Brazilian educational context: a literature review}

\begin{abstract}
This article aims to present, through a bibliographic revision, how occupational therapists have organized their discussions on the use of assistive technology resources at the school environment. An active search around this theme was performed in different databases, Scientific Eletronic Library Online (Scielo); Virtual Health Library (Bireme); Revista de Terapia Ocupacional da Universidade de São Paulo; Cadernos de Terapia Ocupacional da Universidade Federal de São Carlos, for the period between 2005, to the first trimester of 2013. The result showed the systematization of eleven studies related to assistive technology, occupational therapy, the educational context, and educational inclusion. The analysis revealed that occupational therapists worry about the need to educate teachers to use the assistive technology resources in the process of educating disabled students. The number of works found is still small considering the demand of the assistive technology and education area. There is a greater number of works about research reports, and fewer works about the theoretical foundation of assistive technology and its relation with Occupational Therapy in the school environment.
\end{abstract}

Keywords: Mainstreaming, Self-help Devices, Occupational Therapy.

Autor para correspondência: Carla Cilene Baptista da Silva, Departamento de Saúde, Educação e Sociedade, Universidade Federal de São Paulo, Rua Silva Jardim, 136, Vila Mathias, CEP 11015-020, Santos, SP, Brasil, e-mail: carlaci@gmail.com

Recebido em Nov. 26, 2014; $1^{\text {a }}$ Revisão em Maio 4, 2015; $2^{\text {a }}$ Revisão em Maio 26, 2015; Aceito em Ago. 3 , 2015. 


\section{Introdução}

A inserção do Terapeuta Ocupacional no campo da educação, segundo Rocha (2007), se fez através da Educação Especial, em que o trabalho do profissional era voltado ao público com deficiência ou transtorno do desenvolvimento, que estudava em escolas de educação especial, segregadas da rede regular de ensino.

As açóes do Terapeuta Ocupacional nessas instituiçóes consistiam no apoio aos professores mediante as dificuldades apresentadas pelos alunos, como aquelas relacionadas a possíveis adaptaçóes na sala de aula, na estrutura física, na realizaçáo de atividades e orientaçôes sobre o uso de recursos assistivos (ROCHA, 2007).

Com a ampliação das políticas públicas para esta população e das propostas de inclusão dos alunos com necessidades educacionais especiais na rede regular de ensino, o Terapeuta Ocupacional redirecionou o seu contexto e a forma de trabalho para além de uma visão meramente reabilitacional (LOURENÇO; CID, 2010).

O paradigma de inclusão baseia-se na crença em uma sociedade para todos os seus cidadáos - é uma proposta de construção de cidadania. Nesse sentido, inclusão é entendida como um conjunto de ações a serem construídas cotidianamente, objetivando tanto a sensibilização da pessoa com necessidades especiais e de suas famílias sobre seus direitos, deveres e possibilidades como cidadáos, quanto da sociedade que passa a se responsabilizar por todos os seus membros, acolhendo-os de maneira indiscriminada e oferecendo-lhes possibilidades efetivas para a real participação e atuação destes em sociedade (SASSAKI, 1997; MARINS; PALHARES, 2007; ROCHA, 2007; ROCHA; LUIZ; ZULIAN, 2003).

Assim, as intervençóes do Terapeuta Ocupacional não se restringem apenas à criança e ao seu processo de reabilitação, pois suas atuações também ocorrem junto à comunidade escolar e à família, visando a favorecer e melhorar a participaçáo da criança nesse contexto e em todas as atividades que este abrange. As intervençôes podem, ainda, ocorrer através do uso de tecnologia assistiva, na realização de adaptações e reorganizaçôes tanto de ambientes quanto de mobiliários, e nos materiais utilizados pela criança (IDE; YAMAMOTO; SILVA, 2011; DE CARLO; BARTALOTTI, 2001).

Segundo Sartoretto e Bersch (2013), o termo Assistive Technology, traduzido no Brasil como Tecnologia Assistiva (TA), foi criado em 1988, como importante elemento jurídico dentro da legislação norte-americana, conhecida como Public Law 100-407, e que foi renovado em 1998 como Assistive Technology Act de 1998 (P.L. 105-394, S.2432).

A palavra assistiva vem de assistir (que auxilia, assiste e ajuda) e ainda não está incluída no vocabulário português, mas vem sendo utilizada comumente associada à palavra tecnologia. É um termo recente que é empregado para identificar toda a gama de recursos e serviços que colaboram para promover ou ampliar habilidades de pessoas com limitaçóes funcionais, motoras, sensoriais ou mentais e, por conseguinte, promover a independência e a inclusão.

A tecnologia, ao longo da história, vem sendo utilizada para facilitar a vida do homem e passa a ser considerada assistiva quando é utilizada para auxiliar no desempenho funcional das atividades, reduzindo as incapacidades para realização de atividades. Por isso, é comum observar o discurso de que, para as pessoas com deficiência, a tecnologia vai além: é a diferença entre "poder" e "não poder" realizar açóes, uma vez que podem promover a independência, a autonomia e o acesso. Por esse motivo, os recursos tecnológicos especializados ou equipamentos de ajuda estáo sendo utilizados e produzidos com mais frequência nos últimos anos (ROCHA; CASTIGLIONI, 2005).

As tecnologias assistivas, circunscritas na tecnologia, envolvem aspectos mecânicos, biomecânicos, ergonômicos, funcionais, cinesiológicos, éticos, estéticos, políticos, afetivos, subjetivos e, como tal, devem ser compreendidas.

Segundo Rodrigues e Alves (2013), a tecnologia assistiva representa atualmente uma área em ascensão, impulsionada pelo paradigma da inclusão social, que defende a participação de pessoas com deficiência nos diversos ambientes da sociedade. Porém, os autores ressaltam que, apesar da grande demanda da área no Brasil, as pesquisas e os projetos de TA ainda são incipientes e restritos a especialistas envolvidos com pessoas com deficiência.

Algumas políticas públicas brasileiras têm contribuído para gerar demandas de TA em larga escala. Uma delas é a Política Nacional de Educação Especial na Perspectiva da Educação Inclusiva (BRASIL, 2008). As orientaçôes dessa política, com suas normas e orientaçóes para a inclusão escolar de pessoas com deficiência, ressaltam que a plena participação desses alunos pode ser garantida com a presença dos recursos de TA, não apenas no ambiente escolar, mas em todos os processos de aprendizagem desses sujeitos (RODRIGUES; ALVES, 2013).

Nesse sentido, no cotidiano escolar, a TA vem associada à necessidade de adaptação do 
ambiente escolar para o acolhimento da criança com necessidades educacionais especiais, pois a educação inclusiva preconiza mudanças em toda a estrutura educacional, na qual a escola se adequa para receber este aluno, confirmando a ideia de que é o ambiente que deve se adaptar ao aluno e não vice-versa (OLIVEIRA, 2008).

\section{Metodologia}

Trata-se de uma revisão bibliográfica realizada em diferentes bases de dados: Scientific Eletronic Library Online (Scielo); Biblioteca Virtual em Saúde (Bireme); Revista de Terapia Ocupacional da Universidade de Sáo Paulo; Cadernos de Terapia Ocupacional da Universidade Federal de São Carlos, no período de 2005 ao primeiro trimestre de 2013. Partiu-se da proposta de entender os caminhos percorridos pelos terapeutas ocupacionais sobre o uso da Tecnologia Assistiva associada ao contexto educacional brasileiro.

Foram encontrados 11 trabalhos durante esse processo de sistematização dos materiais bibliográficos, a busca foi realizada utilizando os seguintes descritores: Tecnologia Assistiva, Educação Inclusiva e Terapia Ocupacional.

Os resultados foram distribuídos em tabelas, organizados pelo ano de publicação, descrição dos autores, tipo de estudo, título e periódico de publicaçáo.

\section{Resultados}

A publicação dos 11 estudos ocorreu em oito periódicos diferentes, sendo que apenas a Revista Brasileira de Educação Especial teve mais de uma publicaçáo sobre o tema desta revisáo (Tabela 1), correspondendo a $36,3 \%$ da amostra. A maior incidência de publicaçôes ocorreu no ano de 2012, com cinco estudos, seguido do ano de 2009, com três estudos. Os demais anos tiveram apenas uma publicação.

Dos trabalhos encontrados, somente um estudo abordou conteúdos teóricos e epistemológicos sobre a tecnologia assistiva. Os demais foram constituídos de relatos de experiências e de pesquisas. Esses manuscritos apresentam diversas abordagens metodológicas, sendo essas abordagens estudos de casos, pesquisa quantitativa e grupos de formação e atualização profissional (Tabela 2).

\section{Discussão}

O manuscrito mais recente foi o de Plotegher, Emmel e Cruz (2013), que teve como objetivo relatar a utilizaçáo dos produtos assistivos como auxílio no desempenho escolar de alunos com deficiência matriculados na rede regular de ensino de São Carlos-SP. Consiste num relato de projeto de extensão, no qual os estudantes de Graduação em terapia ocupacional realizaram variadasaçôes com alunos com diversas deficiências. Como resultado, destaca-se que as dificuldades apontadas pelos alunos foram comprovadamente amenizadas ou neutralizadas por meio do uso da tecnologia assistiva, além de ter repercutido quanto à percepção dos professores sobre a importância da utilizaçáo dos recursos de TA nas suas salas de aula.

O estudo realizado por Varela e Oliver (2012) visou a compreender a utilização da tecnologia assistiva por crianças com deficiência, na vida cotidiana, sendo a comunidade escolar uma das áreas de desempenho. O objeto de estudo foram cinco crianças atendidas no serviço de atençáo básica em saúde do município de São Paulo, sendo que foram apresentados os recursos utilizados por esse público

Tabela 1. Distribuição dos estudos de acordo com o periódico e o ano de publicação.

\begin{tabular}{|c|c|c|c|c|c|c|}
\hline \multirow{2}{*}{ Nome do periódico } & \multicolumn{6}{|c|}{ Ano } \\
\hline & 2005 & 2009 & 2011 & 2012 & 2013* & Total \\
\hline Cadernos de Terapia Ocupacional da UFSCar. & & & & & 1 & 1 \\
\hline $\begin{array}{l}\text { Revista Brasileira de Crescimento e } \\
\text { Desenvolvimento Humano. }\end{array}$ & & 1 & & & & 1 \\
\hline Revista Brasileira de Educação Especial. & & 1 & 1 & 2 & & 4 \\
\hline Revista Ciência \& Saúde Coletiva. & & & & 1 & & 1 \\
\hline Revista Científica do Unisalesiano Lins. & & & & 1 & & 1 \\
\hline $\begin{array}{l}\text { Revista Informática na Educação: Teoria e } \\
\text { Prática. }\end{array}$ & & & & 1 & & 1 \\
\hline Revista de Terapia Ocupacional da USP. & 1 & & & & & 1 \\
\hline Tecnologia Assistiva (CAT) & & 1 & & & & 1 \\
\hline & 1 & 3 & 1 & 5 & 1 & 11 \\
\hline
\end{tabular}

*Primeiro trimestre de 2013. 
no ambiente escolar, como: cadeira adaptada na sala de aula, andador, cadeira de rodas, triciclo, transporte escolar adaptado e corrimão na escada.

Ferroni e Gasparetto (2012) realizaram um estudo buscando entender a relação com a comunidade e o uso de recursos de TA nas atividades cotidianas, que teve por objetivo conhecer a percepção de alunos com visão subnormal em relação às suas dificuldades visuais.

Ainda no ano de 2012, Gasparetto et al. realizaram uma pesquisa quantiqualitativa com 46 escolares com deficiência visual que estavam matriculados

Tabela 2. A atuação dos Terapeutas Ocupacionais e o uso da tecnologia assistiva.

\begin{tabular}{|c|c|c|c|c|}
\hline Ano & Autor (es) & Periódico de publicação & Título & $\begin{array}{l}\text { Tipo de } \\
\text { estudo }\end{array}$ \\
\hline 2013 & $\begin{array}{l}\text { PLOTEGHER, C. } \\
\text { B.; EMMEL, M. } \\
\text { L. G.; CRUZ, D. }\end{array}$ & $\begin{array}{l}\text { Cadernos de Terapia } \\
\text { Ocupacional da UFSCar }\end{array}$ & $\begin{array}{l}\text { Utilização de dispositivos } \\
\text { assistivos por alunos com } \\
\text { deficiência em escolas públicas. }\end{array}$ & $\begin{array}{l}\text { Relato de } \\
\text { experiência }\end{array}$ \\
\hline
\end{tabular}

M. C.

2012 FERRONI,

M. C. C.;

GASPARETTO,

M. E. R. F.

2012 GASPARETTO,

M. E. R. F. et al.

Revista Brasileira de

Educação Especial

ROCHA,

A. N. D. C.;

DELIBERATO,

D.

2012 SAMESHINA, F. Revista Científica do S.; SILVA, F. R. P. Unisalesiano

2012 VARELA, R. C. Revista Ciência \& Saúde B.; OLIVER, F. C. Coletiva

2011 ALVES, A. C. J.; Revista Brasileira de MATSUKURA, Educação Especial T. S.

2009

GASPARETTO, Tecnologia

M. E. R. F. et al. Assistiva (CAT)

2009a PELOSI, M. B.; NUNES, L. R.

O. P.

2009b PELOSI, M. B.; NUNES, L. R. O. P.

2005 ROCHA, E. F.; CASTIGLIONI, M. C.
Revista de Informática na

Revista Brasileira de

Educação Especial

Assistiva (CAT)

Revista Brasileira de Crescimento e Desenvolvimento Humano

Revista Brasileira de Educação Especial

Revista de Terapia Ocupacional da USP
Escolares com baixa visão: percepção sobre as dificuldades visuais, opinião sobre as relações com a comunidade escolar e o uso de recursos de tecnologia assistiva nas atividades cotidianas.

Utilização de recursos de Tecnologia Assistiva por escolares com deficiência visual.

Tecnologia Assistiva para a criança com paralisia cerebral na escola: identificação das necessidades.

Implementação de Recursos e Procedimentos da tecnologia assistiva no atendimento educacional especializado.

A utilização de Tecnologia Assistiva na vida cotidiana de crianças com deficiência.

Percepção de alunos com paralisia cerebral sobre o uso de recursos de tecnologia assistiva na escola regular.

Uso de recursos e equipamentos de Tecnologia Assistiva na educação municipal, estadual e federal tecnológica.

Formação em serviço de profissionais da saúde na área de tecnologia assistiva: o papel do Terapeuta Ocupacional.

Caracterização dos professores itinerantes, suas ações na área de tecnologia assistiva e seu papel como agente de inclusão escolar.

Reflexões sobre recursos

Estudo teórico tecnológicos: ajudas técnicas, tecnologia assistiva, tecnologia de assistência e tecnologia de apoio.
Pesquisa

Pesquisa

Pesquisa

Pesquisa

Pesquisa

Pesquisa

Pesquisa

Pesquisa

Pesquisa 
em escolas regulares. $\mathrm{O}$ objetivo foi investigar as variáveis: idade, sexo, acuidade visual, visão residual, tecnologia assistiva, informática acessível e interação grupal. Os resultados demonstraram o interesse dos participantes no uso desses recursos em atividades escolares e do cotidiano, possibilitando, assim, a sensibilização da comunidade escolar sobre a eficácia dos recursos de TA com objetivo de auxiliar o aprendizado e o interesse dos alunos nas atividades do ensino regular (GASPARETTO et al., 2012).

O estudo de Rocha e Deliberato (2012) teve como objetivo identificar as necessidades de serviços, recursos e estratégias de tecnologia assistiva para o aluno com paralisia cerebral na escola. Como resultado principal, destaca-se o apontamento das autoras sobre "[...] a importância da capacitação do professor não só para o uso dos recursos, mas para estabelecer as estratégias de uso dos materiais [...]" (ROCHA; DELIBERATO, 2012, p. 87).

Sameshima e Silva (2012) realizaram um estudo que teve por objetivo utilizar um programa baseado nos sistemas suplementares e alternativos de comunicação para capacitar profissionais de uma escola. Foi realizado no município de Lins e teve como objeto de estudo duas professoras especializadas em educação especial. Cabe ressaltar que "[...] os resultados identificados neste trabalho possibilitaram a reflexão sobre questóes imprescindíveis para orientação e capacitação do professor e demais profissionais, quanto ao uso da tecnologia assistiva no contexto educacional [...]" (SAMESHIMA; SILVA, 2012, p. 75).

Alves e Matsukura (2011) realizaram um estudo sobre a percepçáo dos alunos com paralisia cerebral quanto ao uso da tecnologia assistiva na escola regular. Note-se que a potência do trabalho foram os relatos dos próprios alunos acerca do uso desses recursos, podendo assim proporcionar resultados significativos e também observados em outros trabalhos, em que as crianças que faziam uso dos recursos puderam se posicionar quanto às suas necessidades individuais, além de avaliação e percepção da TA.

Gasparetto et al. (2009) tiveram o objetivo de analisar o uso de recursos e equipamentos de Tecnologia Assistiva para auxiliar alunos com deficiência no desempenho das atividades educacionais. Nos relatos, percebe-se que "[...] nem todos os professores que trabalham com alunos com deficiência conhecem e, principalmente, sabem fazer uso do recurso [...]" (GASPARETTO et al., 2009, p. 56).

No mesmo ano, Pelosi e Nunes (2009a) desenvolveram um projeto que teve por objetivo implementar, planejar e avaliar um curso de formação sobre TA, destinado a profissionais da saúde. Os resultados ressaltados neste estudo foram que poucos profissionais (24\%) assinalaram serem capazes de avaliar e determinar as necessidades do aluno, e que menos de $15 \%$ dos profissionais consideraram-se capazes de realizar adaptaçóes para atividades de matemática ou de leitura e escrita.

Os resultados desses estudos se complementam no sentido de promover melhorias em todos os aspectos do uso dos recursos de TA, pois há a necessidade de capacitação dos professores para o uso da TA, mas também "[...] muito se discute em relação à formação continuada de professores e sua realidade de sala de aula e muito pouco se fala sobre a formação dos profissionais da saúde [...]" (PELOSI; NUNES, 2009a, p. 441).

Outro estudo feito por Pelosie e Nunes (2009b) foi realizado junto à Secretaria Municipal de Educação do Município do Rio de Janeiro, no qual se comparava a ação dos professores itinerantes junto aos alunos com necessidades educacionais especiais e apontava soluçôes para favorecer a inclusão dos alunos com deficiência física. Esse estudo indicou que, por mais que as escolas dispusessem de recursos de alta tecnologia, os professores acabavam por não usá-los por falta de instruçáo ou de domínio do manejo.

Por fim, o estudo feito por Rocha e Castiglioni (2005) foi o único a discutir teoricamente o uso da TA, fazendo uma análise quanto ao sentido e à finalidade dos recursos de TA para inclusão de pessoas com deficiência, analisando criticamente a maneira como esses recursos são dispostos, efetivos e satisfatórios para os alunos.

\section{Conclusão}

A partir desta revisão bibliográfica, compreendeu-se que o uso da tecnologia assistiva no ambiente escolar é abordado por diversos terapeutas ocupacionais pela significativa importância sobre o tema, para a comunidade escolar e para crianças com necessidades educacionais especiais.

Os manuscritos encontrados são, em sua maioria, resultados de pesquisas qualitativas, em que a população-alvo prioritária tem sido alunos com necessidades educacionais especiais e professores. A maior parte dos estudos está vinculada a instituiçóes de Ensino Superior. Não foram encontrados relatos das práticas dos terapeutas que não estão vinculados a essas instituiçóes. Apenas um dos trabalhos se direciona para a formação de profissionais da saúde e apenas um estudo discute criticamente e conceitualmente a utilização dos recursos de TA. 
Contudo, esses trabalhos mostram a ação do Terapeuta Ocupacional como um dos agentes modificadores da realidade, muitas vezes, excludente do ensino educacional brasileiro, em que cada profissional demonstra os caminhos que percorre para lutar por uma inclusão escolar com menos barreiras e mais potencialidade.

A escola deve ser compreendida como um ambiente promotor de cidadania, no qual a construção coletiva de um ambiente que esteja apto para a utilizaçáo por qualquer indivíduo faça parte do cotidiano das instituiçôes.

Como elencado pela maioria dos trabalhos, a tecnologia vem aliada a essa possibilidade de mudança e deve ser compreendida e utilizada da melhor forma possível. Para tanto, existe a necessidade da aquisição desses produtos, mas também a formação dos profissionais para a utilização destes, não somente os educadores, mas também os profissionais da saúde que precisam saber avaliar, para contribuir com o uso efetivo da TA.

O Terapeuta Ocupacional tem sido um dos profissionais com formação e conhecimento para empregar a tecnologia assistiva como recurso no processo de inclusão escolar do aluno com deficiência. As salas de recursos multifuncionais e o atendimento educacional especializado presente nas escolas abrem as portas para parcerias importantes no desenvolvimento e na implementaçáo de novas práticas no cotidiano escolar. Porém, o Terapeuta Ocupacional deve se preocupar com a ideia de que os recursos de tecnologia assistiva devem se reduzir às dificuldades individuais. É preciso ir além e fazer com que a utilizaçáo dos recursos em TA sejam acessíveis aos alunos com necessidades educacionais especiais, às famílias e à toda comunidade escolar. Concordamos com Rocha e Castiglione (2005), quando as autoras dizem que os recursos tecnológicos não são em si mesmos facilitadores ou dificultadores dos processos de inclusão escolar. É preciso, em vez disso, tê-los aliados à satisfação e à realizaçáo pessoal dos indivíduos e dos grupos sociais aos quais pertencem.

\section{Referências}

ALVES, A. C. J.; MATSUKURA, T. S. Percepção de alunos com paralisia cerebral sobre o uso de recursos de tecnologia assistiva na escola regular. Revista Brasileira de Educação Especial, Marília, v. 17, n. 2, p. 287-304, 2011. http://dx.doi.org/10.1590/S141365382011000200008 .

BRASIL. Instituto de Tecnologia Social. Tecnologia assistiva nas escolas: recursos básicos de acessibilidade sócio- -digital para pessoas com deficiência. São Paulo: ITS Brasil, 2008.

DE CARLO, M. M. R. P.; BARTALOTTI, C. C. Terapia ocupacional no Brasil: fundamentos e perspectivas. São Paulo: Plexus, 2001.

FERRONI, M. C. C.; GASPARETTO, M. E. R. F. Escolares com baixa visão: percepçáo sobre as dificuldades visuais, opinião sobre as relaçōes com comunidade escolar e o uso de recursos de tecnologia assistiva nas atividades cotidianas. Revista Brasileira de Educação Especial, Marília, v. 18, n. 2, p. 301-318, 2012. http://dx.doi. org/10.1590/S1413-65382012000200009.

GASPARETTO, M. E. R. F. et al. Uso de recursos de tecnologia assistiva na educação municipal, estadual e federal tecnológica. In: BRASIL. Subsecretaria Nacional de Promoção dos Direitos da Pessoa com Deficiência. Comitê de Ajudas Técnicas. Tecnologia assistiva. Brasília: Corde, 2009. p. 41-58.

GASPARETTO, M. E. R. F. et al. Utilizaçáo de recursos de tecnologia assistiva por escolares com deficiência visual. Informática na Educação: teoria e prática, Porto Alegre, v. 15, n. 2, p. 113-130, 2012.

IDE, M. G.; YAMAMOTO, B. T.; SILVA, C. C. B. Identificando possibilidades de ação da terapia ocupacional na inclusão escolar. Cadernos de Terapia Ocupacional da UFSCar, São Carlos, v. 19, n. 3, p. 323-332, 2011. http://dx.doi.org/10.4322/cto.2011.005.

LOURENÇO, G. F.; CID, M. F. B. Possibilidades de açáo do terapeuta ocupacional na educação infantil: Congruência com a proposta da educaçáo inclusiva. $\mathrm{Ca}$ dernos de Terapia Ocupacional da UFSCar, São Carlos, v. 18, n. 2, p. 169-179, 2010.

MARINS, S. C. F.; PALHARES, M. S. Educação inclusiva: relato de uma experiência a partir da visão dos gestores municipais. Cadernos de Terapia Ocupacional da UFSCar, São Carlos, v. 15, n. 1, p. 69-89, 2007.

OLIVEIRA, A. A. S. Adequações curriculares na área da deficiência intelectual: Algumas reflexôes. In: OLIVEIRA, A. A. S.; OMOTE, S.; GIROTO, C. R. M. Inclusäo escolar: as contribuiçôes da educação especial. São Paulo: Cultura Acadêmica, 2008. p. 129-154.

PELOSI, M. B.; NUNES, L. R. O. P. Formação em serviço de profissionais da saúde na área de tecnologia assistiva: o papel do terapeuta ocupacional. Revista Brasileira de Crescimento e Desenvolvimento Humano, São Paulo, v. 19, n. 3, p. 435-444, 2009a.

PELOSI, M. B.; NUNES, L. R. O. P. Caracterização dos professores itinerantes, suas ações na área de tecnologia assistiva e seu papel como agente de inclusão escolar. Revista Brasileira de Educação Especial, Marília, v. 15, n. 1, p. 141-154, 2009b. http://dx.doi.org/10.1590/ S1413-65382009000100010.

PLOTEGHER, C. B.; EMMEL, M. L. G.; CRUZ, D. M. C. Utilizaçáo de dispositivos assistivos por alunos com deficiência em escolas públicas. Cadernos de Terapia 
Ocupacional da UFSCar, São Carlos, v. 21, n. 1, p. 3542, 2013. http://dx.doi.org/10.4322/cto.2013.006.

ROCHA, E. F. A Terapia Ocupacional e as açóes na educação: aprofundando interfaces. Revista de Terapia Ocupacional da Universidade de São Paulo, São Paulo, v. 18, n. 3, p. 122-127, 2007.

ROCHA, E. F. E.; CASTIGLIONI, M. C. Reflexões sobre recursos tecnológicos: ajudas técnicas, tecnologia assistiva, tecnologia de assistência e tecnologia de apoio. Revista de Terapia Ocupacional da Universidade de São Paulo, São Paulo, v. 16, n. 3, p. 97-104, 2005.

ROCHA, A. N. D. C.; DELIBERATO, D. Atuação do terapeuta ocupacional no contexto escolar: o uso da tecnologia assistiva para o aluno com paralisia cerebral na educação infantil. Revista de Terapia Ocupacional da Universidade de São Paulo, São Paulo, v. 23, n. 3, p. 263273, 2012.

ROCHA, E. F.; LUIZ, A.; ZULIAN, M. A. R. Reflexôes sobre as possíveis contribuiçóes da terapia ocupacional nos processos de inclusão escolar. Revista de Terapia
Ocupacional da Universidade de São Paulo, São Paulo, v. 14, n. 2, p. 72-78, 2003.

RODRIGUES, P. R.; ALVES, L. R. G. Tecnologia assistiva - uma revisão do tema. Revista Holos, Natal, v. 6, p. 170-180, 2013.

SAMESHIMA, F. S.; SILVA, F. R. P. Implementação de recursos e procedimentos de tecnologia assistiva no atendimento educacional especializado. Revista Cientifica do Unisalesiano, Lins, v. 3, n. 6, p. 69-78, 2012.

SARTORETTO, M. L.; BERSCH, R. Assistiva - tecnologia e educação. Disponível em: <http://www.assistiva. com.br>. Acesso em: 10 set. 2013.

SASSAKI, R. K. Inclusão: construindo uma sociedade para todos. São Paulo: WVA, 1997.

VARELA, R. C. B.; OLIVER, F. C. A utilização de tecnologia assistiva na vida cotidiana de crianças com deficiência. Ciência \& Saúde Coletiva, Rio de Janeiro, v. 18, n. 6, p. 1773-1784, 2012.

\section{Contribuição dos Autores}

Todas as autoras contribuíram com todas as etapas de elaboração da pesquisa e redaçấo do texto.

\section{Notas}

${ }^{1}$ Este estudo compóe um Trabalho de Conclusão de Curso intitulado: O mapeamento dos recursos e serviços de tecnologia assistiva nas escolas municipais de Santos-SP, desenvolvido para a finalização do Curso de Bacharelado em Terapia Ocupacional pela Universidade Federal de São Paulo - Campus Baixada Santista. 